microscope was given freely, and with that instrument the relative displacements of the star images on the eclipse plate and on one of the comparison plates were measured very accurately. These measurements were corrected for differential refraction, differential aberration and proper motion, and then reduced by the method of least squares. In doing so, the so-called "Entartung" problem discussed by Dr. Freundlich was not touched, and the usual method of six plate constants and Einstein constant was adopted. The observation equations run as follows :

$$
\begin{aligned}
& A+C x+D y+F x^{2}+G x y+E \frac{R \odot}{r} \frac{x}{r}=\Delta x, \\
& B+C y-D x+F x y+G y^{2}+E \frac{R \odot}{r} \frac{y}{r}=\Delta y .
\end{aligned}
$$

Here $x, y$ are in the increasing directions of right ascension and declination. By forming the normal equations, the six plate constants $A, B, C, D, F, G$ and Einstein constant $E$ were calculated, with the following results :

$$
\begin{array}{llll}
E=2 \cdot 13^{\prime \prime} & \ldots & \ldots & \text { Eclipse Plate-No. 115, } \\
E=1 \cdot 28^{\prime \prime} & \ldots & \ldots & \text { Eclipse Plate-No. 119. }
\end{array}
$$

The details will in due course be published in Vol. 18, No. 1 of the Japanese Journal of Astronomy and Geophysics.

Astronomical Institute,

Tôhoku Imperial University, Sendai.

June 20 .

\section{Evaporation of Water through Multimolecular Films}

Hedestrand $^{1}$, Rideal ${ }^{2}$ and Langmuir ${ }^{3}$ have shown that unimolecular films generally do not reduce evaporation from water surfaces into air to an appreciable extent, the reason being that the evaporation resistance (the reciprocal of the evaporation rate) of unimolecular films is of the same order as the evaporation resistance due to collisions of the evaporating molecules with air molecules and subsequent deflection to the water surface. The only exception is cetyl alcohol, which shows appreciable reduction; we found that octadecyl alcohol behaves similarly to cetyl alcohol.

F. Sebba and H. V. A. Briscoe ${ }^{4}$ have recently shown that unimolecular films may produce substantial reduction when they are compressed. They have also investigated a thick film of lubricating oil showing the first interference colours and found a reduction of only a few per cent. However, with films of much greater thickness they found appreciable reduction.

We have investigated the evaporation through layers of $0 \cdot 5-5 \mu$ thickness of paraffin oil, which were obtained by the aid of spreading agents. The research was undertaken with a view to practical application in the protection of dams, etc., in the arid areas of the interior of Australia. The laboratory conditions had, therefore, to be similar to natural conditions, and it was for this reason that evaporation in open Petri dishes was investigated. No particular difficulty is experienced in obtaining a film of about 1-2 $\mu$ thickness which will reduce evaporation by 50-60 per cent. The most successful films are those obtained from dilute solutions (for example, $1: 50$ ) of boiled linseed oil, stand oil, terebinth oil, or high boiling fractions of eucalyptus oil in white paraffin oil. On the other hand, simple fatty acids and alcohols, added to paraffin oil, may cause initial spreading, but the films are unstable and collect into lenses after some time. Lubricating oils also do not generally spread well, although they no doubt contain 'amphipathic' molecules as a consequence of oxidation of paraffins.

Particularly high reductions (up to 99 per cent) have been obtained with thin films $(0 \cdot 5-1 \cdot 0 \mu)$ of certain high boiling fractions of the neutral oil of vertical retort tar. All these observations relate to films on neutral water.

The problem of the stability of these multimolecular films of hydrocarbons, spread by amphipathic molecules adsorbed in the interface, is still obscure. The films deteriorate with time under present experimental conditions. This is probably due to dust settling on the surface. Addition of dust, unless it be purely siliceous, always reduces the effectiveness of the film. There are indications of chemical changes (for example, polymerization) of the spreader, especially in the case of linseed oil and stand oil. However, it is doubtful whether they are responsible to any considerable extent for the deterioration, for no such deterioration is found within two weeks with paraffin - stand oil films investigated in a desiccator, if the film thickness is greater than $1 \mu$. Thinner films, however, show deterioration even under these conditions. We are at present examining the question of stability more closely and we intend to investigate the thermodynamic conditions directly by determination of the spreading coefficients.

The influence of winds on multimolecular films is interesting. It has been found by experiments in a wind tunnel that the reduction of evaporation in a wind is greater than in still air. This apparently surprising result is, however, readily understandable, since the wind increases the rate of evaporation from the free surface more than from a film-covered surface. In other words, the air resistance is considerably lowered by the wind, whereas the film resistance is but little affected. This holds, of course, only so long as the continuity of the film is not seriously affected by the wind. Under laboratory conditions, the film remained effective even when air was blown directly against the surface by a fan. Under natural conditions, on the other hand, the position is more unfavourable as a consequence of the great areas involved. During experiments on a dam of approximately 300 square yards area, it was found that on windy days the film was blown right into a corner, although it usually spread again after the wind had subsided. The main difficulty with respect to dams is, however, due to the short life period (a few days) of the films under natural conditions, most probably as a consequence of the action of dust and suspended material.

We are greatly indebted to the trustees of the Science and Industry Endowment Fund of the Council for Scientific and Industrial Research of Australia for a research grant.

A. R. DOCKING.

E. Heymann.

LuCY F. Kerley.

Chemistry Department, K. N. MoRtensen.

University of Melbourne. May 31.

${ }^{1}$ Hedestrand, G., J. Phys. Chem., 28, 1244 (1924).

' Rideal, E. K., J. Phys. Chem., 29, 1585 (1929).

- Langmuir, L., and Langmuir, D. B., J. Phys. Chem., 31, 1719 (1927).

- Sebba, F., and Briscoe, H. V. A., J. Chem Soc., 106 (1940). 